\title{
Römische Ritornelle.
}

Die nachstehenden 4 Io Ritornelle sind einer umfangreichen, durchaus unter dem musikalischen Vortrag in den 7oger und 8oger Jahren angelegten handschriftlichen Ritornellensammlung entnommen, die dem Herausgeber der Rom. Zeitschrift zur Auswahl übergeben wurde. In den bekannten Büchern von Blessig, Tigri, Marcoaldi, Bernoni u. a. und in der vor Kurzem erschienenen Scelta dei migliori Stornelli amorosi cantati dal popolo italiano von A. Salani (Firenze 1888) gedruckte Stücke wurden, auch wenn die sprachliche Form Verschiedenheiten zeigte, bei Seite gestellt, um das Neue besser hervortreten zu lassen. Beibehalten wurden die römischen Wortformen, die Freiheiten der Versbehandlung, die volkstümlichen Konstruktionen, wie sie der Vortrag darbot, der hier weder verbessert werden sollte, noch verbessert werden konnte, da ursprünglichere reinere Fassungen bis jetzt nicht bekannt sind. $\mathrm{Zu}$ einzelnen Ritornellen sind Varianten am Fufse der Seiten nachgewiesen. Die Mannigfaltigkeit des Inhalts widerstrebte einer der üblichen Gruppierungen nach dem Grundgedanken. Die ersten 127 Nummern wurden erheblich früher aufgezeichnet als der Rest. Rom und Umgebung (Trastevere, Campagna) lieferten mir für beide Abteilungen den Stoff.

1.

Voglio cantare,

Quattro ritornelli voglio dire,

La mi compagna mi risponderd.

2.

Ecco, compagna mia, che t' ho risposto,

Questo ritornello, che $t^{\prime}$ ho detto;

Non so, se l' ho detto a genio vostro.

3.

Amore mio, quanto bello siete

Agl' occhi miei, quanto a genio annate;

Se siete piccolino, crescerete.

Fior di pisello!

4.

Che avete, amore mio, che state giallo,

Vi ha fatto male l'aria di Castello. 
Amore mio,

5.

Non piglia li fiori da gnessuno,

Se voi il garofoletto, te lo dò io.

Fior di canna!

6.

Come volete, ch' jo la notte dorma,

Si sempre penso a voi, core di mamma.

7.

Lo mio amore, che si chiama Checco,

Lo tiengo arritrattato a capo a letto,

Venitelo a vedè, che bel moretto!

8.

Lo mio amore, che si chiama Carlo,

Venitelo a vedè, se quanto è bello,

Lo tiengo arritrattato ner ventaglio.

9.

Lo mio amore si chiama Pasquale,

Gli ho fatto le calzette, e non le vole,

Le voglio andà a buttà in mezzo del mare.

10.

Cupido vi tiro le frezze al sole.

O, Dio del Cielo, quanto fece male,

Fece scoprì l' inganni dell' amore.

II.

Angelo ve chiamate, o che ber nome!

Quattro angeli dal cielo fate calàne

Colle vostre dolcissime parole.

Quanto sei bella!

12.

Te ce vorrebbe una rosa per spalla,

Un mazzo di fiori a quella faccia bella.

13.

Quanto sei bella, Dio ti benedica!

Pare, che ti ha dipinto Santo Luca,

Santo Luca e Santa Margherita.'

Lo mio amore, che si chiama Nino, Venitelo a vedè, se quanto è caro, $\dot{E}$ il meglio tulipano del giardino.

Alla viola!

15.

Chi te se goderà la prima sera

$\mathrm{Li}$ cuscinetti e le bianche lenzuole!

1S. u. No. 401 Variante. 
Fior di riso!

16.

Boccuccia risarella, occhio pietoso,

Boccuccia risarella de paradiso.'

17.

Tutte le catene di Nerone,

Nessuna mi ha potuto incatendne.

Solo che la catena del mio amore.

Bella zitella!

I8.

L'occhio ve ride, e la bocca ve parla,

Il sole ve fà nisconnarella.

Fior de cardo!

19.

Che odore de garofalo che sento!

Sard l' amore mio, che me stà accanto.

20.

Alzando gli occhi al cielo vidi stellato, In mezzo ci era il piede di Cupido, Era di rose e fiori incoronato.

21.

E lo mio amore stà lontano tanto, Me manda li saluti dallo vento, lo lo vento lo ringrazio tanto.

22.

Lo mio amore stà in cima una vigna, Me manda li saluti da una stella; Povero amore mio, chi se lo piglia!

23.

Quanti saluti manda l'amore mio,

Per quante spighe stanno al campo di grano, Per quanti campanelli fa lo lino.

24.

Andetti al giardino a coglie un fiore;

Vidi il bello mio tra le viole, Credevo ch' era la sfera del sole.2

25.

Lo mio amore, che si chiama Pietro, Non si chiama Pietro, che ho sbagliato, Si chiama Federico, e non lo nego.

26.

Fior de patate!

Insegnatemi la via delle palude,

Che lo mio amore me s'è fatto frate. 
Peppetto vojo,

27.

Credessi da magnd pane coll' ajo,

L' insalata senza aceto e olio.

28.

Fior di cannella!

Si diventi 'na candida stella,

lo te dichiaro per prima damigella. 5

Cupido vacce

29.

De quel sangue portàne tre gocce,

Digli, che moriro nelle sue braccie.

Fior d' aneti!

30.

Giacchè i nostri cuori si so sdegnati,

Voi ve ne andate a Napoli, ed io a Velletri.

$3 \mathrm{I}$.

I calzolari puzzano di pece,

I giardinieri odorano di rose,

E lo mio amore de viole accese.

32.

I calzolari addopreno er cortello,

I carrettieri i fazzoletti al collo,

E lo mio amore li fiori al cappello.

Amore mio!

33 .

Pe una mancanza, che ha fatto sto seno,

Siete partito senza dimmi addio.

Fiore di rose!

34 .

Che serve, che mettete tante scuse,

Che per amore se fa tutte cose.

Fior di limone!

35.

Er limone è agro, e le fronde so amare;

So più amare le pene d'amore. ${ }^{2}$

36.

Avete l'occhio nero e brillantino;

Fate calà gl' amanti da lontano,

Massimamente quelli da vicino. ${ }^{3}$

37.

Avete l' occhio nero, e me guardate,

Andate dicendo, che non me volete,

Io non voglio a voi, se me pagate.4

I Vgl. Blessig No. 165.

Vgl. Tigri, Cantis S. 325, 45 .

2 Vgl. Blessig No. 35; Tigri'S. 371, 437.

Vgl. Blessig No. 47². 
38.

Avete l' occhio nero, e siete trista,

Capate l'insalata alla finestra,

Volete fa l' amore senz' esse vista. ${ }^{1}$

39.

Avete l' occhio nero, e il petto bianco:

De quà e di là due lampade d'argento,

Se non piglio a voi, divento matto.

Albero pinto!

40.

Il bene, che ti ho voluto, me ne pento,

Il cuore mio è reale, il vostro è finto.

Fior di radice,

$4 \mathrm{I}$.

Stò in collera con voi, non fo più pace,

Stò in collera con voi, e campo felice. ${ }^{2}$

Fior di cannei!

42.

Mannaggia l'ossa, di quando ti amai,

So iti tutti in fumo $i$ tuoi.pensieri.

Occhi tiranni!

43.

Me hai fatto perde tanti belli sonni,

$\mathrm{E}$ da dormì con voi mi so mill' anni.

44 .

E lo mio amore, che de quà non c' ène!

Vacce Cupido mio, vallo a chiamàne,

Fatlo passà pell' arco de Novene.

Fior di caffe,

45 .

Non vi posso lascid, o dio che sarà,

Che fra me e voi qualche cosa c'e.

46.

Se me date un bacio, me lo piglio;

Se me ne date due, meglio che meglio;

Se me ne date tre, in braccio ve piglio.

47.

Sete il meglio frutto del giardino,

E la briccocoletta del fruttarolo,

Quanto m' andate a genio, cuore mio!

Fior de canna!

48.

Sete la pecorella della vigna,

Sete bambaciona de tata e mamma!

1 Vgl. Blessig No. 277.

Zeitschr. f. rom. Phil. XIII.
2 Vgl. Tigri Cantis S. 359, 331. 
Fior de ragusta!

49.

Quando l' acqua del mare diventa pasta, Allora si troverd una donna giusta.

50.

Quando l' acqua del mare diventa inchiostro, Voglio scrive uną lettera al mi ragazzo, Glie voglio mandà a dì, se è vivo o morto.

Fior di bambace!

51.

Voglio andà all' inferno à fàmme coce, Come li tre fanciulli alla fornace. 1

Vatte a fà frate! 52.

Vattene al convento delle Rete, Dove c' è l' abbondanza delle rape.

Vado per mare!

53 .

Fra la rena me lo persi il cuore,

Lo cerco, e non lo posso ritrovare.

In mezzo al mare 54 .

So tutte bajocchelle nove nove, So tutte zitellucce da maritare.

55.

M' affaccio alla fenestra e vedo il mare, Tutti li bastimenti vedo venire;

Quello del mio amore poco può stare.

56.

Mi affaccio alla fenestra e vedo il porto, Tutti li marinari portano el lutto, Segno che lo mio amore sard morlo.

Marinarello!

57.

Per lo mare vai navigando,

Porte me un saluto a quel figlio bello.

58.

Quanto so belli gli uomini moretti, Specialmente quelli giovanotti, So tanti mazzi di garofoletti.

Odio che pena!

59.

Ho visto lo mio amore alla lontana, Non gli ho potuto dà la buona sera.

1 Vgl. Blessig No. 301. 
60.

Te do la buona notte e vado via:

Me comandate niente, anima cara,

Figlia del Gran Sultano della Turchia!

61 .

Ve do la buona notte e passo ponte,

Ve vengo a riveri, stella galante,

Porti la luna in petto, e il sole in fronte.

62.

Ve do la buona notte, se la volete;

Sennò, la butto pe ste cantonate;

Domattina la ricoglierete.

63.

Al Piemonte c' è una guerra forte, Tutti li giovanotti se vanno a batte,

Ste ragazzette glie danno la morte. ${ }^{1}$

Fior d' agrume!

64.

Il mio amore al Piemonte vo' andare,

Se lui ce va, io me butto a fiume.

Fior d'erbetta!

65.

La gente diranno: povera ragazza,

S'è ita a buttà a fiume pe 'na sciocchezza.

66.

Uno, due e tre, poche parole.

Palazzo fabbricato in mezzo al mare,

Colonna che sostiè questo mio amore.

67.

Santa Maria Maggiore è tutta d' oro.

Tu canti le canzone, io l'imparo,

$\mathrm{Tu}$ butti li sospiri, ed io m' accoro.

68.

Io me ne voglio annd

Mo me ne vado sotto al Colonnato di S. Pietro,

Sotto al Colonnato ti vedo a fa.

Alla bellona!

69.

Mi, ha detto lo mio amore stamattina,

Sta sera ce vedemo pacioccona.

di canna

70.

La canna piccolina è tenerella;

Questo lo dico a voi, cuore de mamma.

1 Seitenstück bei Tigri, Canti' S. 341, 177. 
Fior di canna!

71.

Se volete sto cuore, ditelo a nonna, Che nonna la farà contenta mamma.1

72.

Amore mio, levame una voja, Porte me alla machia a fà la legna, Nel canneto a fà la canna foglia.

Fior di mentuccia! 73.

Pigliate lo schioppetto, annate a caccia!

Portate l' uccelletto a Barberuccia.

Me chiamo Gesso,

74 .

Con una mano scrivo e l' altra scasso,

Non $\mathrm{m}$ ' annate a genio, ve lo confesso. ${ }^{2}$

Fior di canna!

75 .

Pigliate carta, calamaro e penna,

Per scrive le bellezze de sta tiranna.

Fior di fico!

76.

Pazienza, se $\mathrm{m}$ ' avete abbandonato.

Chi cade in povertà, perde ogni amico.

Viole gialle!

77 .

Guardate, si che razza di zitelle, $\mathrm{Si}$ arrubano gli amanti fra compagne!

Fior di nocchie!

78.

Sentite questo cuore, come batte, Pare un martello, quando da le botte.

79.

Lo mio amore si chiama Rocco.

La tramontana l' ha pelato tutto, Col pelo io ce ho fatto el manicotto.

80.

Lo mio amore si chiama quattro bajocchi,

Quando cammina, inciampica alli sassi, Quando discorre, ciancica li gnocchi.

Fiore di rapa!

$8 \mathrm{r}$.

Vedete che bell' aria à questa pupa, Che pare l'aria del giardin del papa. 
82.

Avete gli occhi neri come coccole,

L' avete rotondetti come fiaccole,

L' avete ritondetti come boccole!

Fior di gramiccia!

83.

Questa ragazza mi parea bonaccia,

Adesso vedo, poichè me l'impiccia.

84.

Io benedico il fior di bergamotta;

La moglie troppo grassa mi ributta,

Ma la voglio un tantino ciaccarotta.

85.

Che serve, che ci fate la zerbina,

Se siete entrata nella quarantina,

E la casa Grimaldi è a voi vicina!

86.

Amore mi ha il cervello sconcertato, Nè più distinguo il vino dall' aceto,

$\mathrm{E}$ prendo per arrosto il pandorato.

Fior d'arancio!

87.

La povera ragione diventa un cencio, Quando d'amore è grande lo sbilancio.

88.

L' amore cresce fino alla follia.

L'ira della ragion perde l'idea;

Si pasce d' ambedue la gelosia.

89.

Meglio è soffrir d' un' uomo l' ingiustizia, Che riportare allettamento e grazia

Da femmina guidata da malizia.

Fior di scopa!

90.

Fate la spiritosa, e siete sciapa,

Fate la bella vita, e siete topa.

- 9 r.

Ho scritto una canzona delicala, E per mandarla alla mia dolce vita, $\mathrm{Ci}$ ho fatto una cornice inargentata.

Fior di serpollo!

92.

Avete il finocchietto nel capello;

$\mathrm{E}$ và spuntando qualche grinza al collo. 
Fior di cedrato!

93.

Con quella lingua, che sta sempre in moto, Mettete sotto sopra il vicinato.

Io benedico il fior di portogallo;

Fulmine e donna è un paragone bello;

Che l' una e l' altra tirano al metallo.

Fior di basilico!

95.

E pieno di perigli il mar che valico,

E la vita e la morte stanno in bilico.

Fior di cetrolo!

96.

Parete una linguattola di palo;

$\mathrm{E}$ siete tonda come puntarolo.

97.

Io benedico il fiore dell' indiviola,

Ha sempre del rammarico chi sciala;

E chi pena sovente, ci consola.

Fior di brugna!

98.

La mia signora è donna di montagna;

Ma non lo vuol sentire, e ci s'ingrugna.

99.

Un giorno la pittura assai fioriva,

Adesso in decadenza si trova,

Sol è cresciuta sulla carne viva.

100.

Fior di malva!

Non so, dóve troviate, chi v'assolva,

Che fate la pupazza, e siete calva.

101.

Che sard mai quell' uomo, che si rammenta

Della primiera gioventì consunta,

E che di qualche cosa non si pente!

102.

Io benedico il fior di cipolletta,

Perchè la mia signora dे alguanto matta;

Glie l' ho detto per parte di una staffetta.

Fior di castagna! ro3.

Venite ad abitare nella vigna;

Che siete una bellezza di campagna.

Fior di noccbia!

ro4.

Me l' avvisava il gracchiar della cornacchia,

Che la mia bella donna mi infinocchia. 
105.

Escan di notte fuor tutte le stelle,

Perchè stan chiuse allora le pupille,

Chi son di lor più scintillanti e belle.

I06.

Le stelle dei vostri occhi han gelosia,

Che voi l'aprite sulla luce coa;

$E$ allor le stelle se ne vanno via.

Fior di paglia!

107.

Tuttị dicendo van, ch' è buona figlia;

Ma v'è il rovescio poi della medaglia.

108.

$\dot{\mathrm{E}}$ venuta una barca di fianchetti:

Le giovinotte l'an comprati tutti,

Sino le donne delli vicoletti.

Fanno le donne de' monti le spazzine,

Solo col proveder le nostre ciane,

Di veli, nastri, fiocchi e fettuccine

I 1 o.

Ogni ragazza cova il suo genietto, $\mathrm{E}$ per quanto dimostri il viso brutto, Pure si trova il suo cacazibetto.

Fior di gazzia!

III.

Dopo che miro quella bèlla idea, Io non patisco più d'ipocondria.

Fior di cotone!

112.

Erano le mantiglie cappuccine,

Adesso è gesuita il mantiglione.

113.

Chi troppo tende l' arco, al fin lo strappa,

E se la tua severita sia troppa,

Il discipolo tuo di man ti scappa.

Fior di bieta!

II4.

La cappicciola in oggi è poco usata,

$E$ van le cuoche e le scuffiare in seta.

115.

Di mezzi busti è giunto un navicello:

Il nome non lo so di chi spedillo,

$\mathrm{Ma}$ il vero negoziante è farfarello.

Fior di crescioni!

I16.

Han perduto il cervello li Romani,

Che de cavalli portano i fibbioni. 
117.

Quanti color portate in capo e in seno; $\mathrm{Ci}$ avete il giallo, il rosso ed il turchino, E mi parete voi l' arcobaleno.

Fior di salce!

118.

Guardati dalla vita troppo dolce ;

Che più fastidio ti darà la falce.

Fior di oliva!

119.

Chi a la moglie inquieta, ha fatto l' uovo; E un' uomo mezzo morto e mezzo vivo.

Fior di erbetta!

$$
120 .
$$

La virtù raminga e derelitta

Abita in una inospite isoletta.

Fior di cedro!

I2I.

Pigrizia e povertà vanno d' accordo;

Che la terra non frutta all' infingardo.

122.

La tigre delle belve è la più fiera,

Ma d'ogni femminile creatura

Voi siete al mondo la più gran ciarliera.

Fiore di grano!

$$
\text { I23. }
$$

Avete un natural cosi bonino,

Che Dio ne guardi ogni fedel cristiano.

124.

Una donna, che marcia in guardinfante,

E meglio essere gialla e scolorita,

Che aver dipinto l' una e l'altra gota.

Impiccolite un po' quelli fianchetti, Che la gente vi burla in questi motti; E la mula, che porta i bariletti.

Fior di giunchiglio!

126.

Giacchè mi fate sempre mangiar l'aglio, State da me lontano mezzo miglio.

Fior di canna!

127.

Non criticar l' onore della donna, Che è un cristallo, subito. che appanna. 
128.

Fior di bambace!

In cielo c' è una stella, che riluce;

$\grave{\mathrm{E}}$ segno, che mio amore vuol far pace. ${ }^{1}$

O dio del cielo!

129.

Mi s' è preso collera davvero,

E da rifarci pace, non lo spero.

130.

Io voglio andar alla matrice, Signora madre mia, dateve pace, Piglio un matricianello e campo felice.

131.

Lo mi amore $m$ ' ha mandato una lettera, Colle sue proprie mani mi ha scritta, Dentro c' era scritto, che son moretta.

132.

Io ho mandato un' altra a lui,

Che se li piglia a far tutti sti guai;

Se son moretta, non farò per lui.2

133.

Io benedisco Piazza Barberina,

Il Tiritone e tutta la fońtana;

Quanto mi piace il nome Clementina!

Io benedico la casata Chigi,

La benedisco con tutti li paggi,

Quanto mi piace il nome di Luigi!

135.

Io benedisco. la casa Torlonia,

La benedisco con tutta la sala, Quanto mi piace il nome Agrippina!

136.

Avessi la battecca del commando, Tutti li scultori allo sprofondo, Solo Federico vi raccommando.

Fior di more!

137.

Lo mio amore si chiama Salvatore, Salvatorello, salva me sto core!

138.

E mi amore, che si chiama Annibale,

Venite a veder, quanto è amabile

Da lasciar andar non è possibile.

1 Vgl. Marcoaldi, Canti S. 114, 63.

2 S. u. No. 394-95 Variante. 
139.

Lo mio amore riccio di natura

E di veluto porta la camiciola;

Lo voglio amar, finchè il mondo dura. ${ }^{1}$

O dio, o dio!

140.

Pòssi morir la moglie di Matteo!

Matteo è bello, e me lo sposo io.

Voglio pigliar marito, e voglio a voi, Non me ne curo da star negli guai, Come ce sto io, ce starete voi.

142.

Voglio pigliar marito, lo voglio matto, Se non ha il cervello, io lo metto, O puramente io lo levo affatto.

Voglio pigliar marito, lo voglio vecchio, Non me ne curo di fargli il pan cotto: La prima notte lo butto dal letto.

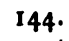

Quanto voglio ben a ste morette; Quelle di quindici anni e dieciasette A far l'amore son briccocolette.

Fior di creta!

Ve son venuto a far la serenata, Se no' m' amate voi, chi se ne lega?

Bella, bella non ve se può dine;

Nemmeno brutta ve se può chiamàne, Sete una zitelluccia cosi cosine.

A li limoni!

147.

Lasciate li passar, che so scultori, E del giardino so li meglior fiori.

Quanto m' importa,

$$
148 .
$$

Se Federico ha gl' occhi di gatta;

Gl' occhi neri il somaro li porta

Vgl. Tigri, Canti3 S. 323, 28. 
Screpantello, mettete la fascia!

Me lo so trovo un' amante capoccio, A sti paini je sputamo in faccia.

150.

A Roma, che se fanno li bicchieri,

E vonno far amore $i$ bicchierari,

L' amore è fatto per i carrettieri!

A Roma che se fanno pile e piatti,

Li muratori vanno per i tetti,

Vanno appresso all'onto come i gatti!

152.

T' aspetto sul cantone a Ave Maria,

Quanto te dico una parola sola;

Quando vedemo mamma, scappamo via.

153.

T' aspetto sul cantone a mezz' ora di notte,

Quanto te dicu .'na parola a parte;

Quando vedemo mamma, buona notte!!

Fior d'aglietti!

154 .

La caccarella di sti giovanotti,

La camicia cogli manichetti.

Fior d' aglietto!

155.

E Pio IX è diventato matto,

Perchè ha dato fuoco a tutto Ghetto.

Fior de pisello!

156.

Quando il papa va a Monte Cavallo, Da la benedizione spara Castello.

Fior de nocchia!

157.

Al curato gli rote la perrucca!

Me vuol mandar via dalla parrocchia.

Fior de granati!

158.

Le donne brutte tutte degli preti, Quelle belle de romiti e frati.

159.

In mezzo al mare che c' è 'na bottega, Dentro c' eे 'na donna maritata, Le va vennendo le strenghe di seta.

1 Verschieden davon Tigri, Canti' ${ }^{3}$ S. 333, 104. 
160.

Aprite il tiratore e fate forte,

Pigliate quella lettera, che stà a parte,

Dentro c'è scritto il nome del mio consorte.

I6r.

Aprite il tiratore e fate pianino,

Pigliate quella lettera, che vi vien in mano,

Dentro c'è scritto il nome di Constantino.

162.

Me so mill' anni, che venga natale,

Per farme 'na magnata de torrone,

Per farme 'na bevuta nel boccale.

163.

Non posso più cantar, che non ho voce!

Ho magnato le persiche duraci,

Ce vuol un bicchier di vino e quattro noci.

Fior di cerasa!

164.

Me fate star colla mente confusa,

Mo che me state lontano da casa.

Fior di cerasa!

165 .

Ve do la buona notte, famo la chiusa,

Lasciamo andar i suoni, andamo a casa.

Fior di limone!

$$
166 .
$$

Se qualcheduno avessi a sospettare,

La serenata, la famo a lampione.

Fior d' ortica!

167.

M' hai fatto mozzicar tutte le dita;

M' hai detto da- venir, non sei venuta.

168.

- Lo mio amore, che fa il muratore!

Venite a veder, quanto fa bene:

Prima mette la calcia e poi il mattone.

$$
169 .
$$

Lo mi amore, che fa l' ebanista?

Prima fa li comò, e poi l'allustra,

Dopo fa le tolette e ci si specchia.

170.

Io de saluti te ne mando trenta:

Cinque per guarnir la prima stanza,

Venticinque alla vostra presenza. 
171.

Io de saluti te ne mando nove:

Se non abbasta sta nove, ecco sta nave,

Se non abbasta sta nave, ecco sto cuore.

Ragazze, non pigliate muratori!

Che ve li fanno far i pianti amari,

Ve le fanno perde li colori.

173.

Si piglia un calzolaro faccia serena,

La sera se ne vien di luna buona,

$\mathrm{Ti}$ dice, moglie mia, che vuoi per cena?

174.

Escita la strazzione, escita quattro.

Che serve, che me fate il cascamorto?

Non fate più per me, datevi al gatto.

175.

Escita la strazzione, escita uno.

Sete rimasta colle mosche in mano,

De tanti amanti non ce $n$ ' avete nessuno ! 1

176.

Bella, che abitate al primo piano,

Fate l' amore con quel chiavarino!

Vi manda li biglietti sotto mano.

177.

Per questa strada, che ci tira vento,

C'è 'na ragazza, che mi piace tanto;

Quel vecchiaccio del padre non n'è contento.

178.

Per questa strada ce son li rondoni.

Quando li vedremo gli sposi nuovi?

Dieci na paolata $i$ lanternoni.

Andiedi in Turchia,

$$
179 .
$$

Trovai una Tucherella tanta cara,

Mi fece rinnegar la fede mia.

Alli lumini!

180.

Quando li sentiremo li belli suoni, Fanno la serenata a Pietro Rubini.

181 .

Quattordici bajocchi un falegname,

Non lo sperate ch' abuschi di piùne,

Attacca ricci, ve vuol governàne.

1 Vgl. Tigri, Cantis S. 345, 212. 
Fior de melella!

182.

Me l' ba detto un pittore, che no sbaglia:

La donna piccolina non fu mai bella! 1

Fior di pane!

183.

$S$ ' è fatto il cappelletto de romoschene;

$O$ dio, che camminata che me ce fàne!

184 .

E quanta me ce fa sta brutta mora, Mo che s' è fatto l' abito a campana, Non se può di' mezza parola.

Fior di niente!

185 .

Piglia la conculina dalla fonte,

Te vengo riverir, stella galante!

186.

L'aria degli monti mi da pena, Perchè i' ho la ragazza montigiana;

E me fa strugge come 'na candela.

Pietro, Pietro!

187.

M' hai rubato il cuore, Pietro ladro,

M' hai rubato il cuor, ladruccio Pietro.

188.

In mezzo al mar è 'na spiga, che punge.

Se sei cristiano, seguita la legge;

Se è destino del cielo, non puoi più fugge.

189.

Per questo vicinato ce ne son trène:

La piccola e la grande, chi la vuòne?

La mezzanella lascio per mène.?

190.

In mezzo al mar una spiga di grano. Intorno intorno un bel circhietto d' oro Una, due, tre fuora mi. chiamo.

Fior di canna!

191.

Chi ha bevuto l' acqua di sta cisterna, Una, due, tre, sempre qui torna.

192.

Se morta me vuol vede, piglia 'r coltello;

$\mathrm{Fa}$, come fece il re di Portogallo,

E della vita mia famo macello.

' Vgl. Blessig No. $27^{2}$ und u. No. 387 .

2 S. No. 233 Variante. 
Preziosa gioja!

193.

Fa, come fece la bella Rachela,

Che vinse la battaglia al re di Troja.

194.

Io de ritornelli ne so tanti:

Ce l' ho da carricar sei bastimenti;

Chi ne sa più di me, si faccia avanti. ${ }^{1}$

195.

Io de ritornelli ne so un sacchetto:

Se me li metto in collo, non li posso,

Se me li metto a dir, non li finisco.

196.

Io de ritornelli ne so una brocca:

Vado strillando, chi vuol l' acqua fresca?

Per dar la minchionella son fatt' a posta.

197.

Mi è stata rigalata una bella mela,

Mi l' ha rigalata la vignarola,

La vignarola in petto la teneva.

Fior di fava!

198.

Vignarolo te porta luttina,

$M$ 'è rigalato un fior de palatana.

Fior de cardi!

199.

Mamma non vuol, che bazzichi li birbi,

Che dicono dolori, quant' è tardi!?

200.

Al palazzo del papa le lendiere

$C$ ' $\mathrm{E}$ 'na ragazza, che se sente male,

Perchè 'l ragazzo non vuol più bene.

201.

Al giardino del papa c'è un bel vaso.

Spero da esser io il vostro sposo;

Perchè, bellina, non me date un bacio?

202.

La mia amore se chiama, se chiama -

Non mi ricordo il nome che aveva;

Si chiama gelsomina, ch' è bella rama.

San Pietro è scuro.

203.

Ce vonno cinque cento torcie d' oro;

Se non c' è il mio amore, non c'è nessuno. ${ }^{2}$.

1 Vgl. Tigri, Canti ${ }^{3}$ S. 320, 2.

2 S. No. 256 Variante.

${ }^{3}$ Vgl. Blessig No. 308. 
Sangnuccio dolce!

204.

Non ve fate mangiar più delle pulci;

Senò, la mamma vostra se n' accorge.

Fior di mela!

205.

Annate a casa, che mamma vi chiama;

Mamma vi chiama, e questo core pena. ${ }^{1}$

Fior di cacio!

206.

L'altra sera al vicolo de buccio

Trovai una vecchierella; mi diede un bacio.

Fior d' insalata!

207.

Mi fate star tutta quanta abbattuta,

Perche mi state lontano da casa?

Fior di limone!

208.

Limone, che si spreme nel bicchiere;

La coccia si ripone nel tiratore.

Fior di nocchia!

209.

Date me tempo un' altra mezz' oretta

Annate all' ombra, che 'I sole me scotta.

210.

Ce avete due bellissime pupille!

Ogni guerrier fate abbassar le arme;

Siete la figlia del guerrier Achille.

211.

Venissi allo steccato de carriera, E con un colpo me ferissi, o cara;

A voi se può dir brava guerriera!

212.

Ce avete due bellissimi colori!

Ce si ponno accende i zolfaroli,

Chi vuol le bellezze, venga da voi.

213.

Io me ne voglio andar in cima uno scoglio, Dove li pesci stanno a far consiglio.

Fossi coperto d' oro, non te voglio.

Garofolo, garofolo piantai!

Sempre la mente mia pensa a voi, $\mathrm{E}$ a me non ci pensate mai. ${ }^{2}$ 
Fior de riso!

215.

Mariuccia mia, mi dai bacio,

Boccuccia risarella di paradiso!

216.

Fior de noce!

Con Nina mia faremo pace;

Allora Nina sua sarà felice.

Fior de giojo!

217.

Rusico la favaccia e mangio l' aglio;

Fosti coperto d' oro, non te voglio.

218.

Chi mangia l'aglio, la bocca puzza,

E chi discorre con voi, poco ci acquista.

Domani è festa!

219.

Guarda la mia ragazza, come s' aggiusta;

Se fa le ricette e si mette in finestra!

Domani è festa;

220.

Un bel mazzetto l' ho mannito a posta,

Per rigalarlo alla mia ragazza.

Fior di ricotta!

221.

Mi voglio divertir com' una matta,

Cantando ritornelli fuori di porta.

222.

Non posso più cantar, non ho favella!

Ho mangiato la minestra calda calda,

Ce vuol un bicchier di vino ed una pagnottella.

223.

Alla finestra vostra ce son li vasi, Tutti gli amanti ce si son confusi, A voi si puó dir: zitella quasi. ${ }^{\prime}$

Fior d' arbuccio!

$$
224 \text {. }
$$

Tengo due core legati con un laccio, Il core di Peppetto e di Petruccio!

225.

Eे morto lo mio amore e non ho pianto;

Me lo credevo, ch' era più tormento!

E morto un papa, e se n' è fatt' un' antro.

1 Seitenstück zu Tigri, Canti S. 368, 4II.

Zeitschr. f. rom. Phil. XIII. 
Fior di giojo!

226.

Date me la mano, quando ballo;

Quando ballate voi, la mano voglio.

Fior di lino!

227.

Agli paini je si da la mano,

Agli minenti je si dice addio.

Fior di patate!

228.

Magnate, non me dite: favorite?

Queste creanze che v' ha, imperate.

Sora Maria!

229.

Pentitevi, pentitevi che è ora!

L' avete fatta grossa la pazzía.

Sor' Onorata!

230.

Volete, che vi porti alla commedia?

Vi piglio un palchetto di facciata.

$23 \mathrm{I}$.

Affacciati alla finestra a darme gusto,

Se non te puoi affacciar, te compatisco.

Te mancano le vesti, la camicia e il busto!

Fior di canna!

232.

Piccolinella mia, piccolinella,

Quando cammini, il petto te balla!

Per questo vicinato $c^{23}$ 'd 'na bella, Quando passa lid, nessuna guarda, Dietro je le fanno la minchionella. ${ }^{1}$

234.

Al vicolo del Cinque ce ne son trène,

La 'piccola e la grande, chi le vòne?

La mezzanella la lasso per mene.

235.

Bella, che avete perso interletto?

Per marito l' avete preso un tappo,

Ce vuol la scala per montar a letto!

Fior de limone!

236.

Come lo rigirate tanto bene!

Avete torto e volete aver ragione.2 
Sora Rosa!

237.

Non ve fate venl i frati a casa,

Perchè i frati vonno qualche cosa.

Sora Santa!

$$
238 .
$$

Aprite il zinnalino e abbiate pazienza,

Che dentro vi ci metto la misticanza!

239.

Mi ̀̀ stato regalato un bel trinciante,

Lo tiengo in petto, e mamma non sa niente.

Ne l' ha regalato lo mio amante. ${ }^{3}$

240.

Cupido mio dell' arco trionfale,

Se trovi il bello mio, che fa amore,

Tira li un razzo de fuoco infernale.

Se passate per l' arco della Ciambella,

Vedete lo mio amore, che gioca a palle,

Diteli, che lo saluta la sua bella.

242.

Non la pigliate bianca, che è ciapita, Non la pigliate rossa, che è fuocosa;

Pigliatela moretta, ch' è saporita!

Sor Abbate!

243.

Volete pigliar moglie e non potete,

$\mathrm{C}^{\prime}$ avete le calzette rinnacciate.

244.

Vatene via, cavallo stornello!

Dagl' altri ti fai mettere brighe e sella.

Quando vedi a me, calci mi dai.

C' hai da capitar sotto questa sella

Cogli sproni ti voglio cavalcare.

Cogli sproni d'or, cogli sproni d'argento,

C' hai da capitar, cavallo scontento?

Alla bellona!

246.

A Serafina damo la corona,

E je la damo, de fronde d' uva l'incoronamo.

247.

Je la damo la corona de l'onore, Quella de Constantino l' imperatoré!

1 Vgl. Tigri, Canti ${ }^{3}$ S. 330, 86. 
Fior d' aglietto!

248.

Incoronamo pure il gobbetto.

Fior di nochia!

249.

Se so seccati alberi alla macchia,

Così si seccherà la lingua vostra.

Alla viola!

250.

Chiamate me li frate della Scala,

Che lo mio amore ha perso la parola. -

251.

Che serve, che da qui voi ce passate,

Intanto la ragazza non ce l' avete,

Le suole delle scarpe vi lograte.

Dillo, che hai?

252.

Vieni alla vigna mia, e dimmi, che vuoi,

I meglior frutti te je piglierai.

253.

Amore mio, $i$ vostri non vonno

Le mie; non si sa, che diavolo hanno.

Siemo contenti, io e voi, e loro che vonno.

Ce so li tessitori de Campidoglio,

Che stanno a lavorar per la fame, che hanno, E tirano la spola a rotta de colli.

Come mai

255.

Pasqua l' hanno presa li gipdei;

Pace tra me e voi non si fa mai!

Fior de cardi!

256.

Mamma non vuol, che bazzichi li birbi,

Perchè li birbi vanno a casa tardi.'

Fior di rampazzo!

257.

Ajuta me, sorella, che sto nel pozzo,

Manda me giù la corda, che me ce attacco.

258.

Comare, vi salutano i piselli,

$\mathrm{Li}$ pulcini con tutti i galli,

Comare, ve ne annate ai pazzarelli.

I S. No. 199 Variante. 
Sora comare,

259.

Ve lo vorebbe chiedere un piacere,

Dormi 'na notte col compare.

260.

Aria agli venti!

Sai, come si governano gli amanti?

Con una rama de ceci spassa tempi.

Fior di niente!

261 .

Volete, che vi pagi il tremolanto?

Basta, che a mamna non je dite niente.

262.

In mezzo al mare che c'è una colonna.

Venite, marinari, a giocà palla,

Chi la vincerà questa vittoria!

263.

Alzando gl' occhi al cielo vidi un comòne

Sopra c' era 'na tazza di caffène

Passo la bella mia, me lo sversòne.

Fior di menta!

264.

Chi dice, che la menta non si pianta?

lo dico, che si pianta e si sementa.

Fior di riso!

265.

Mamma me vuol dare un vecchio bavoso,

lo voglio un giovinotto di bel viso.

266.

Io me ne voglio andar verso la storta

A ritrovar la mia sciaboletta,

Che butta la scianchetta storta.

267.

lo me ne voglio andar verso Livorno,

Dove son le bimbe, che la danno

A chi la buona notte e a chi 'I buon giorno.

268.

A far l'amor con voi, ci vuol moneta,

Ce vonno li testoni de sto papa;

L'orologio d'oro, la fascia di seta!

Fior de more!

269.

Acqua de rondinelle, cerase nere,

Per darle al mio amore, che se ne more.

Fior de more!

270.

Le caccarelle delle zigarare

Portono la triccia a pendolone. 
271.

Alzando l'occhio al cielo, vidi a voi, Subitamente mi innamorai;

Quanto mi piace d' essere di voi!

272.

Alzando l' occhio al cielo, vidi un catino, Dentro c' era il sangue d' un sovrano, C' era il sangue d'Orlando Palatino.

273.

Le stelle dello cielo son quaranta;

Il marinaro dice: conta conta, Quello che cerco io, sempre ci manca.

274.

Per guarir la febbre, ci vuol la china. Per discorre con voi, faccia serena, Bisogna far tre ore di pantomima. 275.

Stelle dello cielo, fate me lume! Insegnatemi la via, dove d'annàne, L' amor è cieco, e non ci vedo lume.

Fior di mortella! 276.

La boccuccia vostra, quando parla, Odora di zucchero e di cannella.

277.

Che serve mamma, che figlia me dite, Intanto le pene mie non le provate? lo fo l'amore, e non me compatite! 278.

Ragazze, non pigliate vignaroli, Che tutto il giorno piantano legumi, La sera 'na pilaccia de faggioli.

Alla Certosa! 279.

Tutti gl' innamorati vanno per casa, Tutti fanno l'amore, nessuno sposa. 280.

Mi voglio far un zinnale de merletto.

La gente mi diranno, chi te l' ha fatto?

Me l' ha fatto il mio amore, sia benedetto! $28 \mathrm{I}$.

Voglio pigliar marito, voglio un barbiere. Non mene curo, se me moro di fame; Me fa portar li ricci a tutte le maniere. 282.

Quanto voglio ben al mio cognato! M' ha imparato a mette la pila al fuoco, NI' ha imparato a cuoce lo stufato. 
Amore, amore!

283.

Se' nato per amore o per amare?

$\mathrm{Se}^{\prime}$ nato per amar sto cuore?

Fior di latte!

284.

Quando mamma te fece, era de notte,

C' erano i pittori a ritrattarti. 1

Fior di ciambrusco.

$$
285 .
$$

Quando so umbriaco, sempre casco,

Biastimo e rifo fuora e guardo lusco.

286.

So nata sfortunata nelle fascie,

Pure la mammana me lo disse:

Povera sfortunata adesso nasce!

287.

Quanto voglio ben a quello core!

Quello che pensieroso mi fa stàne,

Quello che ce l' ha la madre, il padre nòne.

288.

Quanto voglio ben a chi diço io!

Il nome non posso dir davvero,

Lo tengo scritto in mezzo al petto mio.

Agli ruelli!

289.

Sento una pestareggia di cavalli.

E lo mio amore cogli campanelli.

In mezzo al mare,

290.

Dove ce so le trigli e le ceriuole

Quando viene lo mio amore a pescàne!

Alla bellona!

291.

Lo mio amore $\mathrm{m}$ ' ha detto villana:

Son battezzata alla fonte di Roma.

Fior di noce!

292.

A far lo vetturino non sei capace;

Ai cavalli non je sai dar la voce.

A sor paino!

293.

Saccoccia non ci avete, manca un quattrino.

1 Vgl. Blessig No. 85. 
294.

Sor paino con capello bianco, Vi sia levata la pelle dal fianco.

A Sor paino

295.

Le falde le cocimo al tegamino.

296.

Sor paino, con cappello tondo, Voglio veni con voi girando il mondo!

Fior di mortella!

297.

Accanto del vetturino c' è la più bella.

Fatevi indietro,

298.

Che stiamo-sulla piazza di S. Pietro.

Papa Gregorio!

299.

La gabbia con sorcetto moscarolo!

Alla Reale!

300.

L'Ottobre è fatto come carnevale.

301.

Siemo trasteverine tutta foja,

La sapemo far l'arte di boja.

L' arte di boja e l' arte dell' ajutante.

Siemo trasteverine tutte quante.

303.

Siemo trasteverine, e non tremamo

Er cortelluccio in petto, er sercio in mano.

E arrivata una barca di zitelle.

Correte giovinotti a pigliar moglie!

Dieci a bajocco come le mosciarelle.

Voglio cantare,

$$
305 .
$$

E tutto il fiato io voglio fà uscire:

Chi non me può sentire, pòssi crepare.

306.

Chi non può sentì sto mio canto, S' atturino l'orecchio per un momento Fino ch' arriva il prete col olio santo.

307.

Chi non può senti questa mia voce, S' atturino l' orecchio colla pece,

Fino ch' arrivera il prete colla croce. 
308.

Facciate alla finestra, brutta strega, Manico di padella ruzzonita,

Se venghi giù, te voglio dà la paga.

Quanto sei brutta! ${ }^{309 .}$ Ti piglia un dolore,

Te risomigli al peccato mortale,

Dell' inferno sei guardaportone!

310.

State zitta, brutta scanapapere,

Regina delle pecore muggiolose,

Quando parli tu, l' inferno si apre.

Cantate forte!

$$
3 \text { ir. }
$$

Se no 'na pena al cuore ve se mette,

$E$ ve la porterete fin alla morte.

312.

In mezzo al mar un' albero di rose,

Le cime son arrivate alla fornace

Ditelo, bella mia, se vi cuoce.

313.

In mezzo al mar un' albero, che pende:

Le cime son arrivate a ripa grande,

Se l' avete con me, fate l'intende.

314.

Santa Maria maggiore, c' è la salita, All' Aracoli c' è la scalinata.

Sora scontenta, fate la finita!

Alla finestra non ci son sportelli, Di quà di là due belle papagalli :

Grugnaccio spizzicato dagl' uccelli.

Fior d' assogna!

316.

Non tanta chiaccheretta, sora carogna, Annate cercando chi vi gratta la rogna.

Me chiamo schiatta e crepa, e son signora.

Sto nome me l' ha messo la mammana,

Chi vuol male a me, non campi un' ora.

Quanto sei brutta!

318.

Se te butto al mare, la barca scappa,

Arriva la balena e ti ributta. 
319.

Chi vuol male a me, si cacci gl' occhi,

Poi se li faccia alla padella fritti,

Poi me lo mandi a di, quando son cotti.

Mazzaccio giallo!

320.

Che pòssi far la morte, che fece il grillo.

Fu straccinato a coda di cavallo.

Fior de granato

321.

Ce l' hai la coda de paglia, daje fuoco,

In nome tuo, chi l' ha mentovato.

Fior d' aneto!

322.

A dì male di me, vi puzza il fiato, Lavate vi la' bocca coll' aceto.

Siete più bianca, che non è un caldaro;

Siete più rossa, che non è un cetrolo;

C' avete la boccuccia da sommaro.

324.

Mi chiamo Pippo e Peppe, lo sapete;

Dieci a bajocco do le coltellate.

De pugni e schiaffi, quanti ne volete.'

325.

Quanto siete bella a parte dietro!

A parte d'avanti non t' avevo veduto, Pari l' acqua santa di S. Pietro.

Quanto sei bella!

326.

Da lontano mi pari una stella,

$\mathrm{E}$ da vicino un sacco di carbonella.

327.

State zitta, che non sai cantàne,

Piglia la sedia e mettete a sedere,

Piglia la conocchia e mettete a filane.

Passa via!

328.

Chi la conosce, la razzaccia tua!

Prima facevi il ladro e mo la spia.

Fior d' arbuccio!

329.

Delle vostre bellezze me fo un faccio,

Le butto al mondezzajo, e poi ci piscio.

1 Vgl. Blessig No. 286. 
Fior di finocchio

330.

Siete voi, che siete vecchio,

Vene cascato in trediciore in tocco.

Fior d' aglietto!

33 .

Se vi sentite male, andate a letto,

Ve passo la giornata, ve do un pappetto.

Fior de pepe!

332.

Mi è stato detto, che vi c' addannate,

La testa per i spicioli battete.

Fior de more!

333.

Non vi pigliate collera, comare,

Che chi si piglia collera, si more. ${ }^{1}$

Perchè non andante a Valle e non ve vendete?

Pigliate un coltelluccio e ve scannate.

Perchè non te butti

335 .

Dentro lo sciacquatore dei piatti?

Riesci per canali, vai per condotti.

336.

Il bene, che te voglio, te lo dico;

Te vorria veder morto impiccato,

La testa rivoltata per panico.

337.

Se la piglia con me, la piglia male,

Se la piglia con me, pensaci bene,

lo son la sciuma dell'acqua di mare. ${ }^{2}$

338.

E l'acqua della mare è turchinella,

La lingua delle donne cuce e taglia,

Questo dico a voi, bella zitella!

Fior de cavoli;

339.

Pigliamo una carrozza, andamo a Tivoli,

Per discorre con voi ci vuol tre paoli.

Agli ruelli!

340.

Se ti vuoi maritar, perchè non parli?

Te porto dallo stagnaro, e te fo gl' annelli.

1 Vgl. Tigri, Cantis S. 373, 451.

2 Seitenstǘck zu Tigri, Canti ${ }^{3}$ S. 354, 287. 
Fior d' assogna!

341.

Quando vostra madre andette a Bologna, Andette a pigliar rimedio per la rogna.

Fior de Ormo!

342.

Vorria saper le chiacchere, a che stanno;

Chi te fa chiacchera, bocca de forno?

Quanto canti!

343.

Nemmene si avessi gli strumenti,

Te butto a dietro per non cascar avanti.

344.

Me voglio far un zinnale di cutocurno,

$\mathrm{Da}$ piedi ci voglio mette, ti piglia un mal anno,

Si piglia un' anticore, chi sta col grugno.

345 .

Che si' ammazzato, sempre stai allo specchio!

Te spuntano le corna,

Come un' abbacchio.

346.

Dite me, bella, quanti cori ce ne avete,

Che a tutti buona cera mostrate

Solo a me, quando me vedete, la testa voltate.

347.

Vi compatisco, che ragazze siete,

Se non sapete amar, bella, impararete!

Sora Felice!

348.

Vi voglio dir una cosa, che vi dispiace,

Vi siete fatta come un' alice.

349.

Facciaccia verde e pieno di veleno,

Un giorno sentirai, che brutto tono,

Se la linguaccia non te tenghi a freno!

Fior de sale!

351.

Che vai, facendo lingua serpentina,

Che degli fatti miei ne dichi male.

Fior di gazzia!

350.

Mannaccia a tutti i mortacci tua, Quelli de tu padre e de tu zia.

Fior d' anchenne!

352.

Vi pendone le laccie dalle mutande,

$\mathrm{Vi}$ fate minchione dalle zitelle. 
Fior di pepe!

353.

Pepe ve chiedo, pepe me date,

Che bella peparola che ce avete!

354 .

Voglio piglià un bajocco d'aghe e spille;

Te le voglio buttar dietro le spalle:

Amanti come voi trovo mille. 1

A pigliar un scultore, non lo pensate.

Non so bastante le loro monete;

E meglio, che zitelluccia ve ne state.

Fior d' anchenne!

$$
356 .
$$

Ce ne vonno dieci canne,

Per vestire mio amore colle sorelle.

Fior de fica!

\section{7 .}

E tanto tempo, che non l' ho magnata, Voglio farme una ficarolett' amica.

Fior di rampazzo! 358.

La luna sta in mezzo al cielo E sulla testa del mio ragazzo.

359.

Per questa strada ce batte un' orologio;

$\mathrm{E}$ per Gigetto ce vorebbe un bacio,

E per Ninetta una sedia d'appoggio.

Mamma, mamma!

360.

Per marito voglio un suona-tromba,

Tutta la notte va suonà la banda.

361.

Il primo amore fu un caccaro;

Il secondo screpatello vignarolo,

E l'ultimo sarà sto figlio caro.

362.

Un mazzo de garofoli in cantina, Che bella gioventù trasteverina!

363.

Un mazzo de garofoli e viole, $E$ quel paino m' ha rubato il cuore.

364.

In mezzo al tamburello c' è un pupazzo. Evviva Giuseppina col ragazzo!

' Vgl. Blessig No. 52, Tigri, Canti ${ }^{3}$ S. 368, 4 10. 

376.

Bella, che alla finestra ve ne state, La cassa dello petto ve rompete, Vi prego, bella, che ve ce levate!

377.

Tra lusco e brusco e tra le fronde in fresco!

Che avete, bella mia, non vi capisco,

Parlate me italiano e non tedesco!

378.

Bella, che ve ne state dalla fratta!

Non vi fate tirar più la calzetta,

Dopo della calzetta vien la scarpa.

Per dispetto di chi non vorria,

Col mio ragazzo parlo ogni sera;

Parlate, se ce avete gelosia!

380.

Dalla legnara c'è tanta legna,

Gi getto ci la legorono;

La mogliuccia sua se lo ingegna.

Fior de facioli!

381 .

Angelinetta degli cappellari

$\mathrm{Fa}$ l' amore cogli sergiaroli.

Quanto sei nera!

382.

Perchè non dichi a mamma, che te lava.

Con agro di limone e un pò di rena?

Fior d' ormello!

383.

Vedi quel giovinotto, quanto è bello!

Se lo guardi in saccoccia, c' d il coltello.

Fior d' aglio!

384.

Il giovinotto porta il coltello;

Ferma te ricotta, che mo te taglio.

Fior d' erbetta!

385 .

Il ballo sulla piazza, che v' aspetta; Sta sul cantone, batte la cianchetta.

Fior d' erbetta!

386.

Quando lo vedo, ci divento matta,

Il mio ragazzo colla scoppoletta. 
Fior d' erba bella!

387.

Me ha detto un pittor, che non sbaglia

La donna piccinina non è mai bella.

Fior de latte!

388.

Dopo la gelosia pure le batte,

Almeno fossi bella bugiarate.

Alla viola!

389.

Il giorno di S. Pietro scaccio magara, Me fo gli stivaletti e la giacca nuova.

Core tiranno!

391.

Prima me venivi a trovar ogni giorno,

Adesso si vedemo una volta all' anno.

390.

Bella, che abita sotto al tetto,

Il vostro marito è un cornuto contento,

Siemo tutte e due d' un sentimento.

392.

Sonato mezzo giorno vota pile.

Andamo, amor mio, a mangiare;

Dopo mangiato andamo a dormire.

Fior d' erbetta!

393.

La vita della donna è bona tutta

Massimamente quella ciabafetta.

394.

Lo mio amore $\mathrm{m}$ ' ha mandato una lettera, Sigillata con una cerata;

Me m' ha mandato dir, che son moretta.

395.

Io ho mandato un' altra a lui,

Che si li piglia a fà tutt' questi guai;

Se son moretta, non farò per lui. ${ }^{1}$

396.

Io P' ho mandato la risposta,

Che si proveda lui, che io so provista,

Che me son preso un guardian di posta.

Fior di riso!

397.

Apre la finestrella e dentro traso,

A mamma je la famo l' improvviso.

I S. o. No. 128-30 Variante. 
398.

Fior di nocchie!

Se per sorte mammita ti batte,

Pigli te li tutti e buona notte.

Fior di riso!

399.

Boccuccia risarella, da me un bacio!

Boccuccia risarella del paradiso!1

Fior d' argento!

400.

Me son sognato che mi dormivi a canto, Se lo facemi o un sonno contento.

Quanto sei bella, Dio te benedica!

Pare, che abbia dipinto S. Luca

O puramente $\mathrm{S}$. Margherita.2

402.

Voglio buttar una lettera in alto mare!

Nessuna donna te póssi piacere,

Sempre il nome mio pòssi chiamare.

403.

In mezzo al mare, in mezzo alle rovine

Son vivo, me piangete per mortale,

Il male mio un giorno avrà fine.

Andai al giardino pet ricoglie un fiore,

Vidi lo bello mio fra le viole,

E mi pareva la sfera del sole!s

405.

Andai al giardino per ricoglier una rama, Vidi lo bello mio, che riluceva,

E stava tra li fiori di madricala.

406.

Io benedico la menta romana, Quello che si pianta la mattina, E lo mio amor Paolo si chiama.

Fior d' argento!

407.

Piglia una vedovella, e non scaccia tanto; Sarai chiamato padre prima dal tempo.

IS. o. No. 16 Variante.

3 S. No. 24 Variante.
2 S. o. No. 12 Variante. 
408.

A Roma, a Roma le belle romane!

$E$ son più belle le trasteverine,

A rubà cuore so le montegiane.

Fior di nocchia!

409.

Lo mio amore m' ha detto moretta,

Costa piu il caviale che la ricotta.

410.

Se canti in chiesa, come in casa canti,

Povero Cristo, sfortunati i Santi.

\section{Anmerkungen.}

No. 31 Viole accese $=$ Viola di pasquá, Levkoje, also hier frische Levkojen. - 42 mannaggia = Ausruf, etwa „verdammt", "verflucht". - 80 ciancicare gnochi $=$ stottern. - 136 battecca di commando = Zauberstab. 149 paino = gutgekleideter Herr. - 156 spara Castello $=$ Der Schuls Mittags von Castel St. Angelo. - 159 strenghe di seta $=$ seidene Stränge oder Schnüre. -178 paolata $=$ paolo. -181 abuscare $=$ verdienen, ricci $=$ Hobelspähne. - I83 cappelletto de romoschene = langhaariger weicher Filzhut, der noch vor 25-30 Jahren in Rom vom Volk getragen wurde. Die Haare waren so lang, dals man Muster darauf blasen konnte. - 184 abito a campana $=$ glockenförmig (Krinoline). -200 le lendiere $=$ Loggien, Balken. - 217 rusica la favaccia = nagt an schlechten Bohnen. - 238 misticansa = gemischter in der Campagna gesuchter Salat. - 242 ciapita = ist matt, ohne Geschmack. - 243 calsette rinnacciate - gestopfte Strümpfe. 278 pilaccia de faggioli = Topf mit Bohnen. - 285 Ciambrusco $=$ lambrusco. -289 ruello = lustig, heiter. -304 moscerelle = ausgehülste Kastanien, werden auf der Stralse ausgerufen. - 308 russonita = verrostet. 310 scannapapere $=$ Schimpfwort, scannare+papera, pecore muggiolose = rotzige Schafe. - 316 assogna = sugna. - 344 cutocurno = Baumwollenzeug. - 370 circhio $=$ Reifrock. -381 sergiaroli $=$ Strafsenpflasterer. traso $=$ Pt. 2u trarre.

F. Schulzz. 